\title{
Sobre os tipos de Hesperiidae descritos por Roeber (Lepidoptera) ${ }^{1}$
}

\author{
Olaf H. H. Mielke ${ }^{2}$
}

\begin{abstract}
Comments on the systematic position and synonymy of the Hesperiidae described by J. Roeber are presented. The Syn.n. are the following : Jemadia gigantea Roeber, 1925 of Nosphistia zonara (Hewitson, 1866), Pyrrhopyga josepha Ploetz, 1879 of Hegesippe luteizona(Mabille, 1877), Phocides palaemonides Roeber, 1925 of Phocides palemon palemon (Cramer, 1777), Phocides xenocrates Bell, 1935 of Phocides vulcanides Roeber, 1925, Phocides parvus Roeber, 1925 of Phocides pialia pialia (Hewitson, 1857), Thymele aulicus Roeber, 1925 of Astraptes enotrus (Cramer, 1781), Py thonides zonula Mabille, 1889 of Paches loxus loxus (Westwood, 1852), Thespieus chlorocephala Roeber, 1925 of Thespieus lutetia (Hewitson, 1866), Xeniades cecropteroides Roeber, 1925 of Niconiades caeso (Mabille, 1891) and Thracides nanea chiricana Roeber, 1925 of Thracides nanea nanea (Hewtson, 1867). Metron leucogaster leucomelas (Roeber, 1925) is a Comb. n. and a Stat. n. for Zenis leucomelas. Lectotypes are designated for the following species or subspecies: Mimoniades hemitaenia Roeber, 1925, Mimoniades inaequalis Roeber, 1925, Amenis similis Roeber, 1925, Mysoria erythrostigma Roeber, Myscelus nobilis meridionalis Roeber, 1925, Phocides iocularis Roeber, 1925, Phocides parvus Roeber, 1925, Polygonus amyntas pallida Roeber, 1925, Thymele albifasciatus Roeber, 1925, Thymele subfasciatus Roeber, 1925, Thymele aulicus Roeber, 1925, Phyrrhocalles kruegeri Roeber, 1925, Vettius phyllides Roeber, 1925 and Xeniades cecropteroides Roeber, 1925.
\end{abstract}

A presente nota visa esclarecer a posição sistemática dos Hesperiidae descritos por J. Roeber, cujos tipos até hoje eram considerados desaparecidos, dando subsǐdios ao "Checklist" do "Atlas of neotropical Lepidoptera" (Heppner, 1984).

Roeber descreveu, entre os anos de 1925 e 1927, 48 espécies de Hesperiidae neotropicais, muitas não reconhecidas até o presente (EVANS, 1951-1955), principalmente devido ao tipo de descrição e sem qualquer figura.

Em recente visita ao "Staatliches Museum fuer Tierkunde", Dresden, República Democrática Alemã, surpreendi-me ao encontrar 30 dos 48 tipos procurados dentro da coleção Staudinger \& Bang-Haas, adquirida pelo museu em

1 Contribuição n! 611 do Departamento de Zoologia, Universidade Federal do Paraná. Com auxilio do $\mathrm{CNPq}$.

2 Departamento de Zoologia, Universidade Federal do Paraná, Caixa Postal 19020, 81504 Curitiba, Paraná, Brasil. Bolsista do CNPq. 
1961. Os 18 tipos faltantes continuam desaparecidos e provavelmente perdidos pois, como é sabido, Roeber era negociante e vendia tudo, principalmente para colecionadores amadores. Não os encontrei em outros museus europeus. Procurei-os no "Zoologisches Museum der Humboldt Universitaet”, Berlin, República Democrática Alemã, onde está guardada a parte principal da coleção Staudinger e em cuja coleção também suspeitava poder encontrá-los. A identificação dos tipos foi possivel através das etiquetas manuscritas e/ou em comparação com a descrição original. Há, no entanto, algumas discrepâncias, julgadas não relevantes, mencionadas em cada espécie. As etiquetas manuscritas não parecem ser de Roeber (compare a sua caligrafia em HORN \& KAHLE, 1935, pl. XI), porém são todas escritas pela mesma pessoa, inclusive a de Pyrrhocalles kruegeri, única espécie que estava devidamente caracterizada como tipo.

1. Comentários sobre as espécies cujos tipos foram encontrados.

Quando foi necessário, designei um lectótipo, sendo o exemplar devidamente caracterizado, assim como os holótipos, parátipos e paralectótipos.

1.1. Nosphistia zonara (Hewitson, 1866).

Jemadia gigantea Roeber, 1925a: 156, uma fêmea, sem procedência, sem coleção. Syn. n.

Jemadia scomber Druce; Evans, 1951: 52, part., sinônimo incorreto: gigantea Roeber, 1925.

Encontrei o holótipo. Embora seja um exemplar de $7 \mathrm{~cm}$ de envergadura alar, é um sinônimo de Nosphistia zonara e não de Jemadia scomber como o afirma Evans. Possuo outro exemplar fêmea, com a mesma envergadura, procedente do Rio Arajuno, Ecuador.

1.2. Mimoniades sela aequatorea Roeber, 1925. Fig. 1

Mimoniades aequatorea Roeber, 1925a: 90, um macho, Macas, Ecuador, coleção Roeber ("diante de mim").

Encontrei o holótipo. É uma subespécie de Mimoniades sela (Hewitson, 1866).

1.3. Mimoniades sela periphema (Hewitson, 1875). Fig. 2.

Mimoniades inaequalis Roeber, 1925a: 91, três machos, Rio Songo, Bolivia, 750 m, A. H. Fassl leg., sem coleção.

Encontrei um sintipo, designo-o lectótipo. É um sinônimo de Mimoniades sela periphema.

1.4. Mimoniades pityusa (Hewitson, 1857). Fig. 3.

Mimoniades hemitaenia Roeber, 1925a: 91, cinco machos, Ecuador e

Colômbia (dois exemplares do Vale do Magdalena), sem coleção.

Encontrei um sintipo, do Vale do Magdalena, designo-o lectótipo. É um sinônimo de Mimoniades pityusa. 

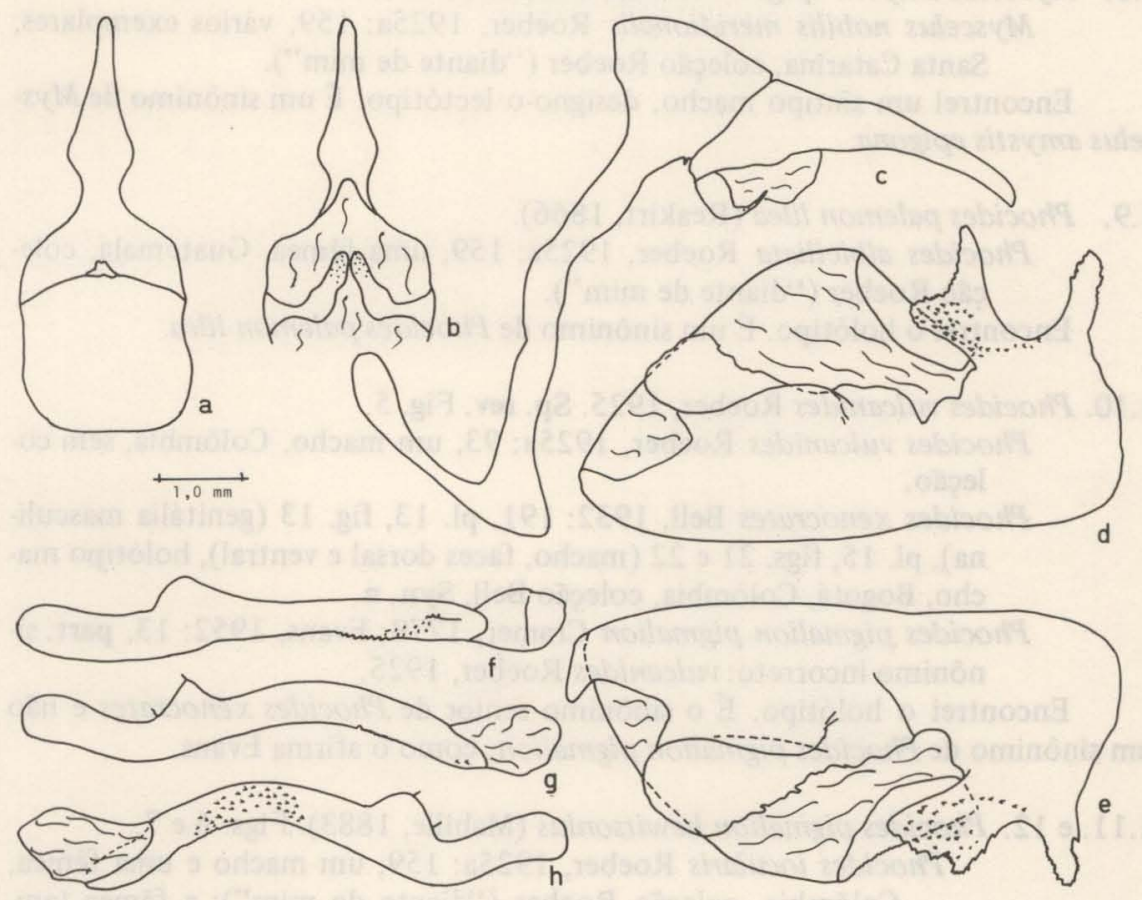

FIG. 1 - Mimoniades sela aequatorea Roeber, 1925. Genitália masculina, holótipo: a - vista dorsal do tegumen e unco, b-vista ventral do gnato e unco, c - vista lateral esquerda do tegumen, saco, unco e gnato, d - vista interna da valva direita, e - vista interna da valva esquerda, f - vista ventral do edéago, $\mathrm{g}$ - vista lateral esquerda do edéago, $\mathrm{h}$ vista lateral direita do edéago.

\subsection{Mimoniades minthe (Godman \& Salvin, 1879). Fig. 4.}

Mimoniades angustifascia Roeber, 1925a: 92, um macho, Macas, Ecuador, sem coleção.

Encontrei o holótipo. É um sinônimo de Mimoniades minthe.

1.6. Amenis pionia pionia (Hewitson, 1857).

Amenis similis Roeber, 1925a: 86, macho e fêmea, sem número de exemplares, Venezuela, sem coleção.

Encontrei um sìntipo fêmea, designo-o lectótipo. É um sinônimo de Amenis pionia pionia.

1.7. Mysoria thasus erythrostigma Roeber, 1925.

Mysoria erythrostigma Roeber, 1925a: 87, sem sexo, sem número de exemplares, Rio Songo, Bolivia, 750 m, A. H. Fassl leg., sem coleção. Encontrei um sintipo macho, designo-o lectótipo. É uma subespécie de Mysoria thasus (Cramer, 1782). 
1.8. Myscelus amystis epigona Herrich-Schaeffer, 1869.

Myscelus nobilis meridionalis Roeber, 1925a: 159, vários exemplares, Santa Catarina, coleção Roeber ("diante de mim").

Encontrei um sintipo macho, designo-o lectótipo. É um sinônimo de Myscelus amystis epigona.

1.9. Phocides palemon lilea (Reakirt, 1866).

Phocides albiciliata Roeber, 1925a: 159, uma fêmea, Guatemala, coleção Roeber ("diante de mim").

Encontrei o holótipo. É um sinônimo de Phocides palemon lilea.

1.10. Phocides vulcanides Roeber, 1925. Sp. rev. Fig. 5.

Phocides vulcanides Roeber, 1925a: 93, um macho, Colômbia, sem coleção.

Phocides xenocrates Bell, 1932: 191, pl. 13, fig. 13 (genitália masculina), pl. 15, figs. 21 e 22 (macho, faces dorsal e ventral), holótipo macho, Bogotá, Colômbia, coleção Bell. Syn. n.

Phocides pigmalion pigmalion Cramer, 1779; Evans, 1952: 13, part. sinônimo incorreto: vulcanides Roeber, 1925.

Encontrei o holótipo. É o sinônimo senior de Phocides xenocrates e não um sinônimo de Phocides pigmalion pigmalion, como o afirma Evans.

1.11. e 12. Phocides pigmalion hewitsonius (Mabille, 1883). Figs. 6 e 7.

Phocides iocularis Roeber, 1925a: 159, um macho e uma fêmea, Colômbia, coleção Roeber ("diante de mim"); a fêmea também como Phocides disparilis (veja a seguir).

Phocides disparilis Roeber, 1925a: 160, uma fêmea, Colombia, coleção Roeber ("diante de mim"); é a mesma fêmea de Phocides iocularis.

Encontrei os sintipos de iocularis. Designo o macho com $50 \mathrm{~mm}$ de envergadura como lectótipo. É um sinônimo de Phocides pigmalion hewitsonius. Encontrei também o holótipo de disparilis (também sǐntipo de iocularis). É um sinônimo de Phocides pigmalion hewitsonius.

\subsection{Phocides pialia pialia (Hewitson, 1857).}

Phocides parvus Roeber, 1925a: 93, dois machos, Brasil, sem coleção.

Syn. $n$.

Encontrei um sǐntipo macho, designo-o lectótipo. É um sinônimo de Phocides pialia pialia.

1.14 e 15. Polygonus leo leo (Gmelin, 1790).

Polygonus (Acolastus) histrio Roeber, 1925a: 161, um macho, Panamá (?), sem coleção.

Polygonus amyntas pallida Roeber, 1925a: 161, sem número de exemplares, Peru, sem coleção.

Encontrei o holótipo de histrio e dois sintipos machos de pallida. Destes, designo o do Peru como lectótipo e o outro, de Lima, como paralectótipo. 


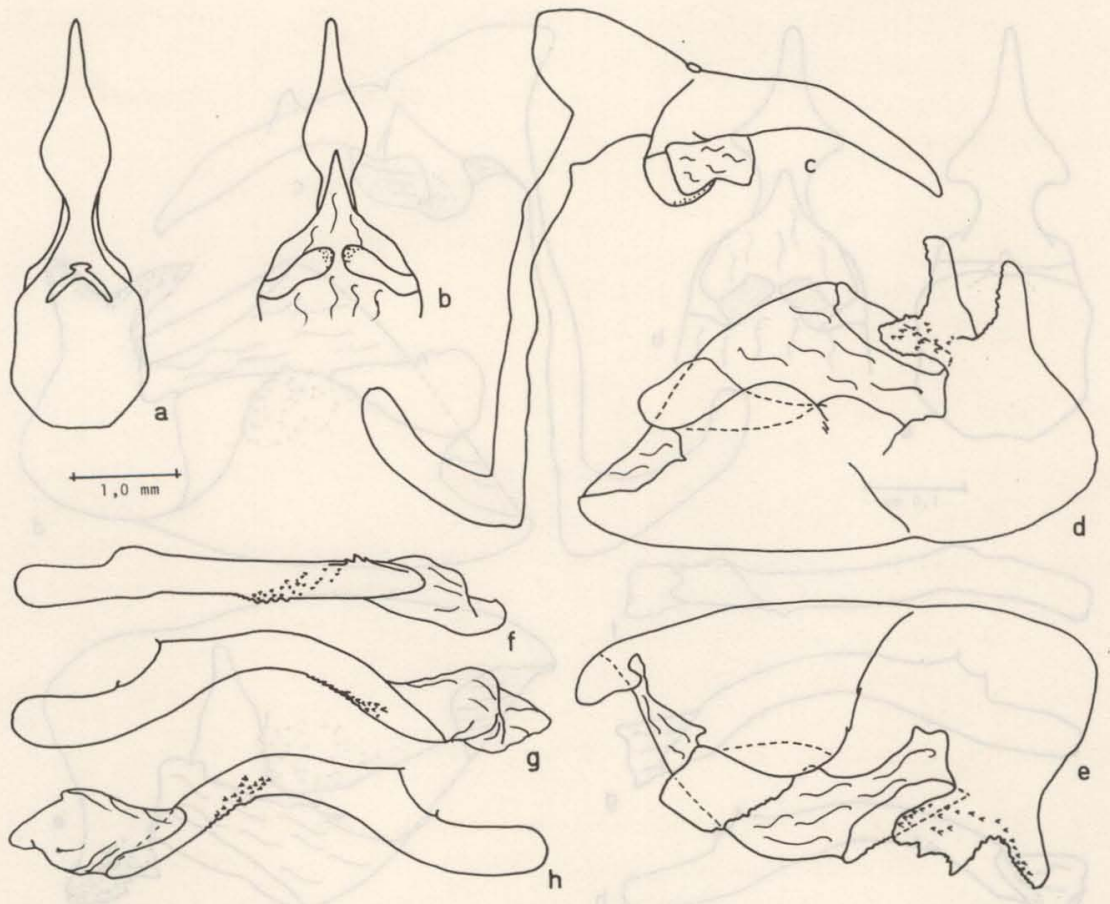

FIG. 2 - Mimoniades sela periphema (Hewitson, 1875), genitália masculina, lectótipo de inaequalis: a - vista dorsal do tegumen e unco, b-vista ventral do gnato e unco, cvista lateral esquerda do tegumen, saco, unco e gnato, $d$ - vista interna da valva direita, e - vista interna da valva esquerda, f- vista ventral do edéago, $\mathrm{g}$ - vista lateral esquerda do edéago, $\mathrm{h}$ - vista lateral direita do edéago.

1.16. Urbanus virescens (Mabille, 1877).

Eudamus chlorothrix Roeber, 1925a: 160, um macho, Huancabamba, Peru Central, 1500 m, A.H. Fassl leg., sem coleção.

Encontrei o holótipo. É um sinônimo de Urbanus virescens.

\subsection{Astraptes fulgerator azul (Reakirt, 1866).}

Thymele albifasciatus Roeber, 1925a: 96, dois machos, Rio Songo, Bolǐvia, 750 m, Fassl leg., sem coleção.

Encontrei os dois sintipos, designo-os respectivamente lectótipo (com o ângulo anal da asa posterior esquerda danificado) e paralectótipo. É um sinônimo de Astraptes fulgerator azul.

1.18. Astraptes naxos (Hewitson, 1867).

Thymele subfasciatus Roeber, 1925a: 96, dois machos, São Paulo, sem coleção. 


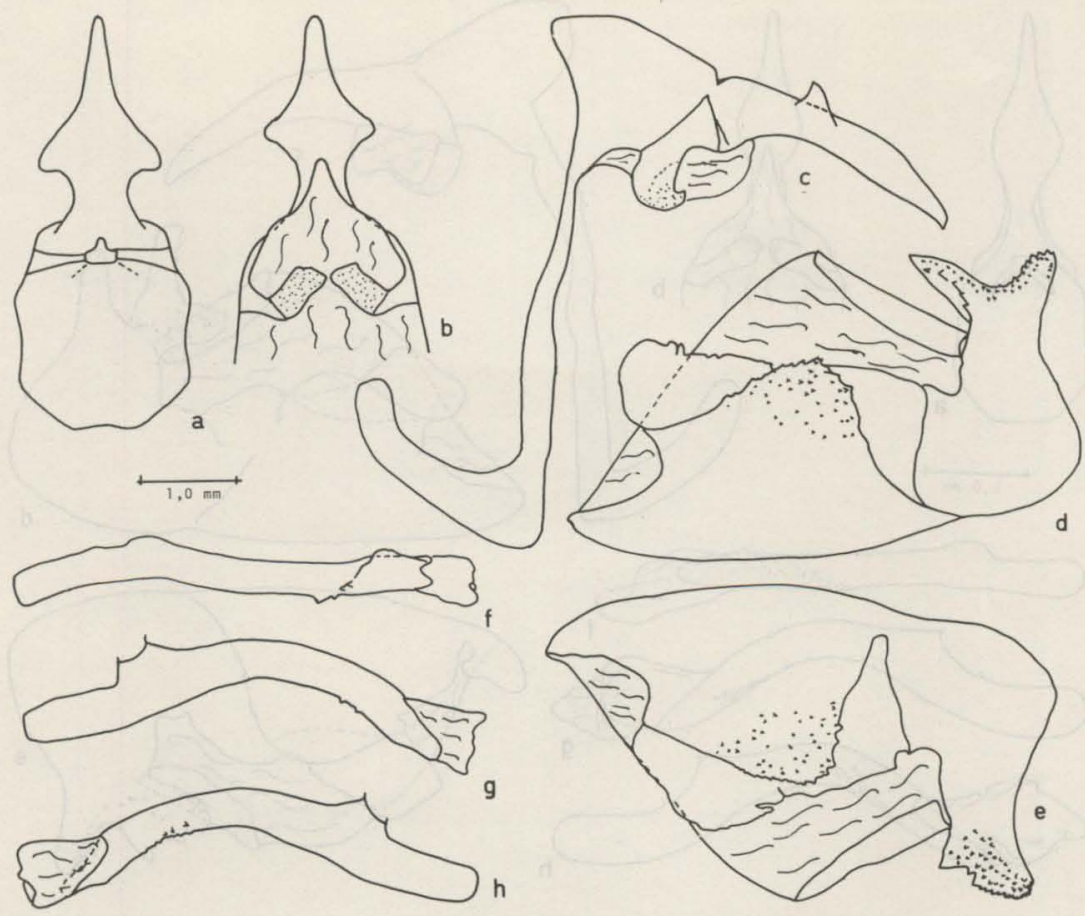

FIG. 3 - Mimoniades pityusa (Hewitson, 1857). Genitália masculina, holótipo de hemitaenia: a - vista dorsal do tegumen e unco, b-vista ventral do gnato e unco, c - vista lateral do tegumen, saco, unco e gnato, $d$ - vista interna da valva direita, e - vista interna da valva esquerda, f - vista ventral do edéago, g - vista lateral esquerda do edéago, $\mathrm{h}$ - vista lateral direita do edéago.

Encontrei os dois sintipos, designo-os respectivamente lectótipo (com a perna anterior direita preparada ao lado da antena direita) e paralectótipo. É um sinônimo de Astraptes naxos.

\subsection{Astraptes enotrus (Cramer, 1781).}

Thymele aulicus Roeber, 1925a: 96, um macho e uma fêmea, Santa Catarina, sem coleção. Syn. $\mathbf{n}$.

Encontrei o sintipo macho, designo-o lectótipo. É um sinônimo de Astraptes enotrus.

\subsection{Paches loxus gloriosus Roeber, 1925. Stat. n.}

Paches gloriosus Roeber, 1925a: 98, um macho, sem procedência, coleção Roeber ("diante de mim").

Paches loxus loxus Westwood, 1852; Evans, 1953: 128,part. sinônimo 

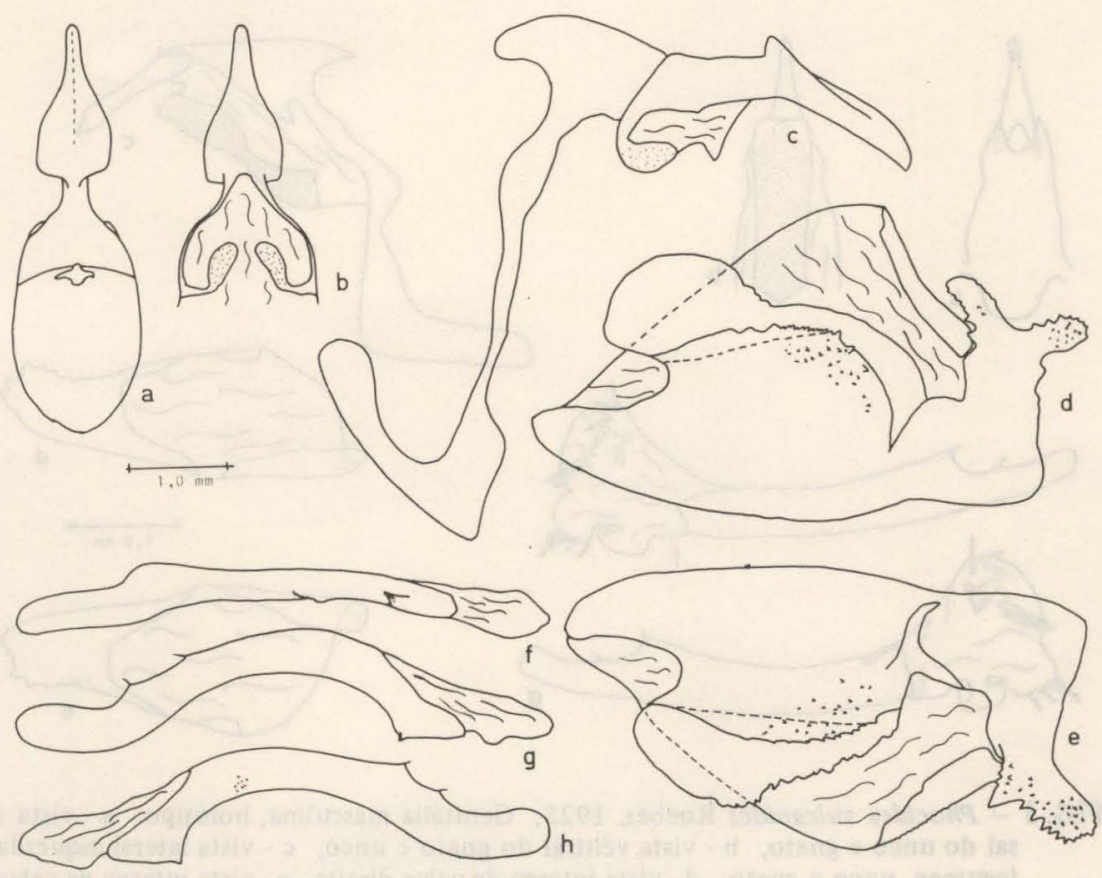

FIG. 4 - Mimoniades minthe (Godman \& Salvin, 1879). Genitália masculina, holótipo de angustifascia: a - vista dorsal do unco e gnato, b-vista ventral do gnato e unco, cvista lateral esquerda do tegumen, saco, unco e gnato, $d$ - vista interna da valva direita, e - vista interna da valva esquerda, f - vista ventral do edéago, $\mathrm{g}$ - vista lateral esquerda do edéago, h - vista lateral direita do edéago.

incorreto: gloriosus Roeber, 1925.

Paches loxus zonula Mabille, 1889; Evans, 1953: 128.

Encontrei o holótipo de gloriosus com a mancha sobre a célula discal e a área adjacente da face dorsal da asa anterior um tanto avermelhada e com as antenas parcialmente partidas, como menciona a descrição original. É a subespécie de loxus que ocorre entre o México e a Costa Rica, mencionada por Evans como zonula, e não um sinônimo de loxus loxus conforme menciona o mesmo autor. O único sintipo de Pythonides zonula por mim encontrado no "Zoologisches Museum dar Humboldt Universitaet", Berlin, é uma fêmea proveniente do Panamá; um sinônimo de Phareas loxus Westwood, 1852, Syn. n.

\subsection{Carrhenes canescens pallida Roeber, 1925.}

Carrhenes pallida Roeber, 1925a: 99, um macho, Rio Grande do Sul, sèm coleção.

Encontrei o holótipo. É a subespécie de Carrhenes canescens (Felder, 1869) do sul do Brasil, Paraguai e Argentina. 

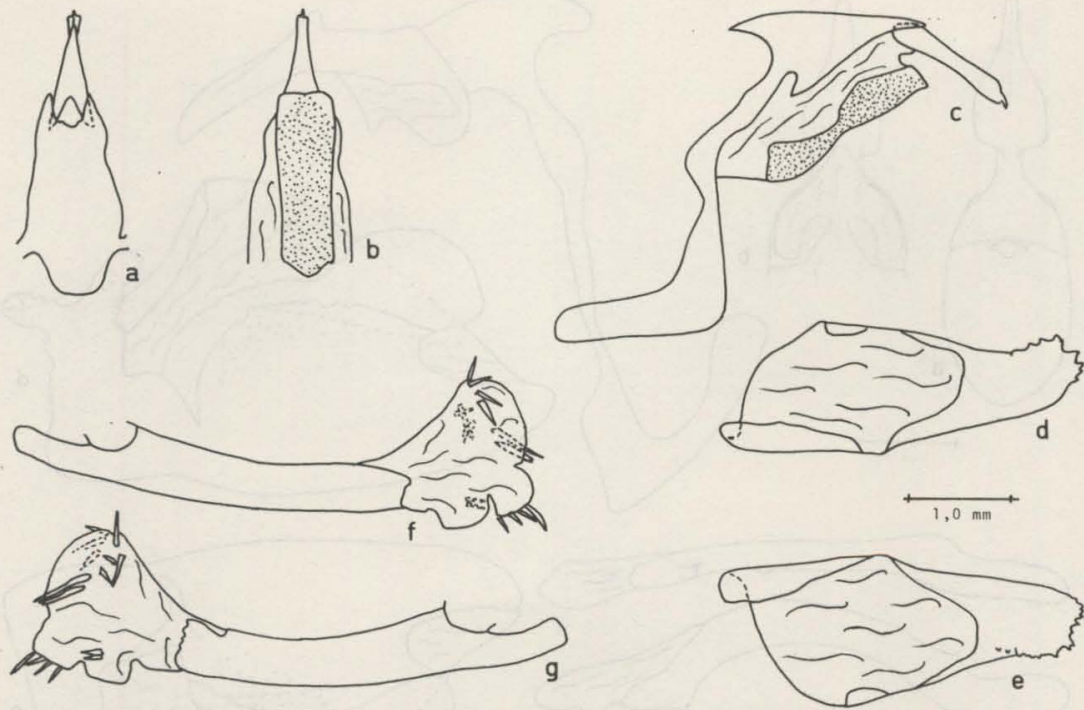

FIG. 5 - Phocides vulcanides Roeber, 1925. Genitália masculina, holótipo: a - vista dorsal do unco e gnato, b - vista ventral do gnato e unco, c - vista lateral esquerda do tegumen, unco e gnato, d - vista interna da valva direita, e - vista interna da valva esquerda, $\mathrm{f}$ - vista lateral esquerda do edéago, $\mathrm{g}$ - vista lateral direita do edéago.

\subsection{Heliopetes laviana laviana (Hewitson, 1868).}

Heliopetes dividua Roeber, 1925a: 99, um macho, rio magdalena, oeste da Colômbia, sem coleção.

Encontrei o holótipo. É um sinônimo de Heliopetes laviana laviana.

1.23. Pyrrhocalles antiqua antiqua (Herrich-Schaeffer, 1863).

Pyrrhocalles kruegeri Roeber, 1926: 32, dois machos, 16-VI-1925, Ilha Santo Domingo, Coleção Krueger.

Encontrei um sǐntipo, designo-o lectótipo. É um sinônimo de Pyrrhocalles antiqua antiqua.

1.24. Vettius marcus marcus (Fabricius, 1793).

Vettius phyllides Roeber, 1925a: 99, um macho e uma fêmea, Santa Catarina, coleção Roeber ("diante de mim").

Encontrei os dois sintipos. Designo o macho como lectótipo e a fêmea como paralectótipo. É um sinônimo de Vettius marcus marcus.

1.25. Perichares seneca seneca (Latreille, 1824).

Lychnuchoides nubilus Roeber, 1925a: 97, um macho, Rio Grande do Sul, sem coleção.

Encontrei o holótipo, uma fêmea (coincide com a descrição) sem abdome. É um sinônimo de Perichares seneca seneca. 


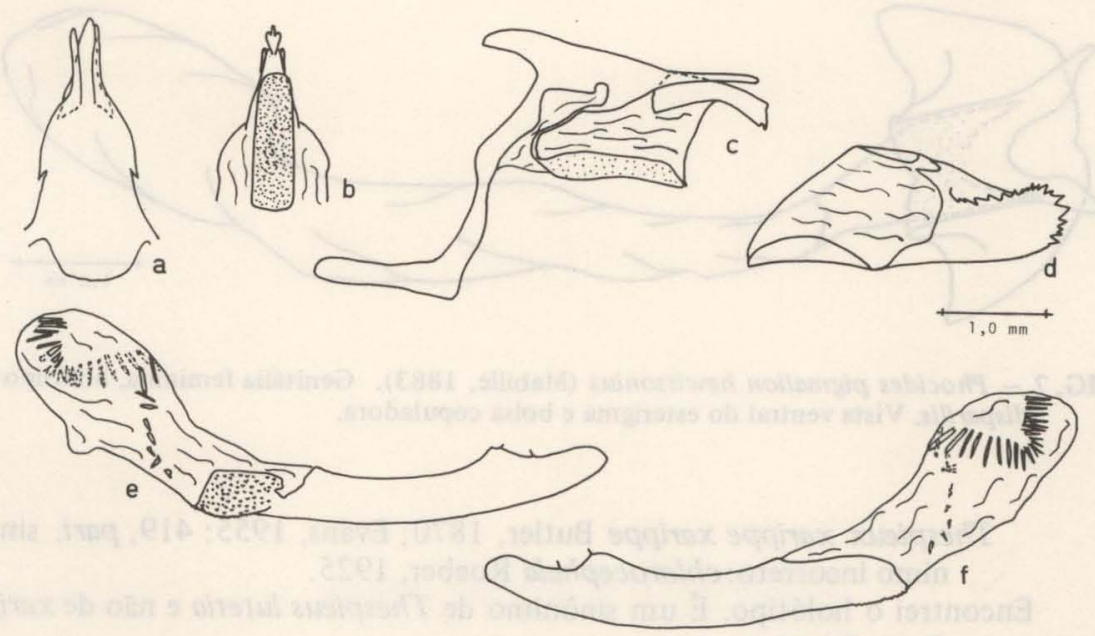

FIG. 6 - Phocides pigmalion hewitsonius (Mabille, 1883). Genitália masculina, lectótipo de iocularis: a - vista dorsal do unco e gnato, b-vista ventral do gnato e unco, c vista lateral esquerda do tegumen, saco, unco e gnato, d - vista interna da valva direita, e - vista lateral esquerda do edéago, f - vista lateral direita do edéago.

1.26. Metron leucogaster leucomelas (Roeber, 1925). Comb.n., Stat. n. Figs. 8-11 Zenis leucomelas Roeber, 1925a: 97, um macho, Rio Grande do Sul, sem coleção.

Cobalus calvina Hewitson, 1866; Evans, 1955:241, part. sinônimo incorreto: ? leucomelas Roeber, 1925.

Encontrei o holotipo, uma fêmea com as antenas incompletas, como descrito, e com a envergadura alar de $28 \mathrm{~mm}$ e não de $22 \mathrm{~mm}$. É uma subespécie de Metron leucogaster (Godman, 1900) do sudeste brasileiro. Deve ser uma espécie muito rara. Possuo um macho e uma fêmea sem procedência, mas provavelmente de Joinville, Santa Catarina, pois foram coletados por K. Schmidt. No American Museum of Natural History, Nova York, há uma fêmea de Hansa Humbold (atual Corupá), Santa Catarina. Não é um sinônimo de Cobalus calvina como o afirma Evans.

Esta subespécie é muito semelhante a Metron leucogaster ambrosei (Weeks, 1906) da Venezuela. A julgar pela figura deste (Weeks, 1911, p. 18, pl. XI, fig. 2 - fêmea dorsal e ventral), leucomelas possue a mancha do espaço 2 da asa anterior maior e retangular e a faixa branca da face dorsal da asa posterior de limite externo uniformemente convexo, enquanto que em ambrosei há uma reentrância no espaço $1 \mathrm{c}$.

1.27. Thespieus lutetia (Hewitson, 1866).

Thespieus chlorocephala Roeber, 1925a: 161, um macho, Brasil, sem coleção. Syn.n. 


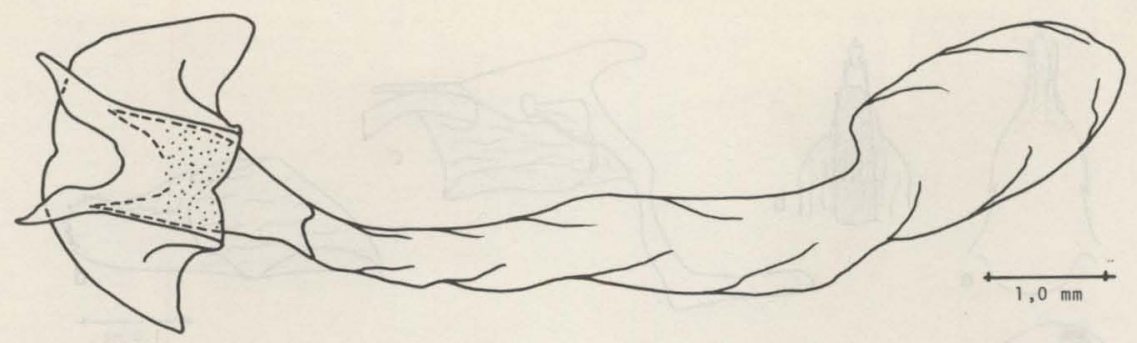

FIG. 7 - Phocides pigmalion hewitsonius (Mabille, 1883). Genitália feminina, holótipo de disparilis. Vista ventral do esterigma e bolsa copuladora.

Thespieux xarippe xarippe Butler, 1870; Evans, 1955: 419, part. sinônimo incorreto: chlorocephala Roeber, 1925.

Encontrei o holótipo. É um sinônimo de Thespieus lutetia e não de xarippe como o afirma Evans.

1.28. Niconiades caeso (Mabille, 1891).

Xeniades cecropteroides Roeber, 1925a: 97, um macho e uma fêmea, Santa Catarina, sem coleção. Syn.n.

Niconiades cydia Hewitson, 1876; Evans, 1955:433, part. sinônimo incorreto: cecropteroides Roeber, 1925.

Encontrei o sǐntipo fêmea, designo-o lectótipo. É um sinônimo de Niconiades caeso e não um sinônimo de cydia, como o afirma Evans.

1.29. Oxynthes corusca (Herrich-Schaeffer, 1869).

Xeniades leucogaster Roeber, 1925a: 98, uma fêmea, Rio Grande do

Sul, coleção Roeber ("diante de mim").

Encontrei o holótipo. É urn sinônimo de Oxynthes corusca.

1.30. Thracides nanea nanea (Hewitson, 1867).

Thracides nanea chricana Roeber, 1926: 26, um macho, Chiriqui, sem coleção. Syn. n.

Encontrei o holótipo. É um sinônimo de Thracides nanea nanea. Este nome foi omitido por EVANS (1955).

2. Comentários sobre as espécies cujos tipos não foram encontrados.

Os tipos das espécies, a seguir listados, não foram encontrados e provavelmente estão perdidos.

2.1. Pyrrhopyge telassina telassina Staudinger, 1888.

Pyrrhopyge telassa var. telassina Staudinger, 1888: 295, pl. 99 (como telassa), machos, sem número de exemplares, Chamchamayo e Bolivia, sem coleção. 

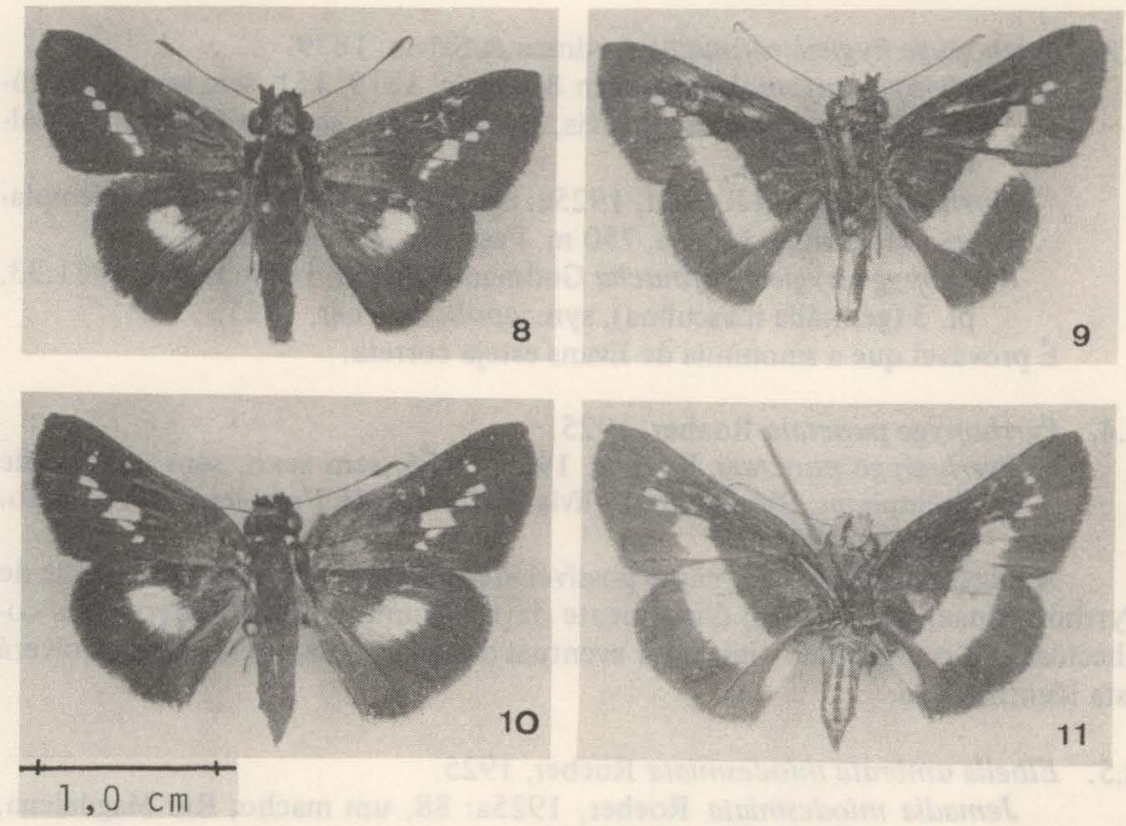

FIG. 8 - 11 -Metron leucogaster leucomelas (Roeber, 1925); 8, Macho, face dorsal; 9, Idem, face ventral; 10, Fêmea, face dorsal; 11, Idem, face ventral.

Pyrrhopyge olivacea Roeber, 1925a: 86 , sem sexo, sem número de exemplares, Peru, sem coleção.

Pyrrhopyge telassina telassina Staudinger, 1888; Evans, 1951:25, pl. 2 (genitália masculina), syn.: olivacea Roeber, 1925.

Tive a oportunidade de estudar os sintipos de telassina no "Zoologisches Museum der Humboldt Universitaet", Berlin e confirmar a identificação de Evans. Só o exame da genitália do tipo ou a eventual designação de um neótipo de olivacea resolverá esta sinonïmia.

\subsection{Pyrrhopyge hygieia hygieia (Felder \& Felder, 1867).}

Tamyris hygieia Felder \& Felder, 1867:506, pl. 70, fig. 1, sem sexo, sem número de exemplares, Nova Granada, Bogotá, Lindig leg., coleção Felder.

Pyrrhopyge phoebus Roeber, 1925a: 86, sem sexo, um exemplar, Vale do Cauca, Colômbia, coleção Roeber.

Pyrrhopyge hygieia hygieia Felder \& Felder, 1867; Evans, 1951:32, pl. 3 (genitália masculina), syn.: bogotana Reakirt, 1868, phoebus Roeber, 1925 .

É provável que a sinonǐmia de Evans esteja correta. 
2.3. Pyrrhopyge hygieia rufinucha Godman \& Salvin, 1879 .

Pyrrhopyga rufinucha Godman \& Salvin, 1879: 151, sem sexo, sem número de exemplares, Bolfvia, Buckley leg., coleção Godman \& Salvin.

Pyrrhopyge apollo Roeber, 1925a: 86, macho, sem número de exemplares, Rio Songo, Bolivia, 750 m, Fassl leg., sem coleção.

Pyrrhopyge hygieia rufinucha Godman \& Salvin, 1879; Evans, 1951:33, pl. 3 (genitália masculina), syn.: apollo Roeber, 1925.

É provável que a sinonĭmia de Evans esteja correta.

2.4. Pyrrhopyge punctata Roeber, 1925.

Pyrrhopyge punctata Roeber, 1925a: 156, sem sexo, sem número de exemplares, Rio Songo, Bolívia, 750 m, A. H. Fassl leg., sem coleção. - Evans, 1951: 22.

A julgar pela descrição, não é possǐvel identificá-la com qualquer espécie de Pyrrhopyginae, no entanto, dificilmente deve ser uma espécie diferente das conhecidas. Só o exame do tipo ou a eventual designação de um neótipo resolverá esta identificação.

2.5. Elbella umbrata miodesmiata Roeber, 1925 .

Jemadia miodesmiata Roeber, 1925a: 88, um macho, Rio Magdalena, oeste da Colômbia, sem coleção.

Elbella umbrata miodesmiata Roeber, 1925; Evans, 1951: 45, pl. 5 (genitália masculina).

A confirmação deste nome só será possivel após o exame do tipo ou a eventual designação de um neótipo.

2.6. Elbella polyzona extrema Roeber, 1925.

Jemadia extrema Roeber, 1925a: 158, uma fêmea, sem procedência, sem coleção.

Elbella polyzona extrema Roeber, 1925; Evans, 1951: 48.

A julgar pela descrição, não deve ser o que Evans pensou, pois o tipo de extrema possui uma envergadura alar de $58 \mathrm{~mm}$ (pode estar errado), enquanto que a subespécie identificada por Evans não passa de $50 \mathrm{~mm}$; outros caracteres, no entanto, a aproximam desta. Somente o exame do tipo ou a eventual desig. nação de um neótipo resolverá esta identificação.

\subsection{Jemadia hewitsonii albescens Roeber, 1925.}

Jemadia albescens Roeber, 1925a: 87, um macho, Macas, Ecuador, sem coleção.

Jemadia hewitsonii albescens Roeber, 1925; Evans, 1951: 53.

A confirmação deste nome só será possĩvel após o exame do tipo ou a eventual designação de um neótipo.

2.8. Mysoria barcastus venezuelae (Scudder, 1872).

Pyrrhopyge venezuelae Scudder, 1872: 67, sem sexo, sem número de exemplares, Venezuela, sem coleção. 
Mysoria caucana Roeber, 1925a: 162, um macho, Vale do Cauca, oeste da Colômbia, sem coleção.

Mysoria barcastus venezuelae Scudder, 1872; Evans, 1951: 67, syn.: caucana Roeber, 1925.

É provável que a sinonimia de Evans esteja correta.

2.9. Hegesippe luteizona (Mabille, 1877).

Pyrrhopyga luteizona Mabille, 1877: 162, sem sexo, sem número de exemplares, México, coleção Mus. Roy. Belg.

Pyrrhopyga josepha Ploetz, 1879: 534, sem sexo, sem número de exemplares, Brasil, sem coleção. Syn. n.

Sarbia amoena Roeber, 1925a: 87, dois machos, estado de São Paulo, sem coleção.

Hegesippe luteizona luteizona Mabille, 1877; Evans, 1951: 62, pl. 7 (genitália masculina), syn.: amoena Roeber, 1925.

Examinei os tipos de luteizona e josepha, respectivamente no Institut Royal de Sciences Naturelles, Bruxelas e no "Zoologisches Museum der Humboldt Universitaet", Berlin. São sinônimos, sendo josepha uma forma negra, isto é, sem manchas amarelas nas asas que ocorre junto com a forma tïpica. É uma espécie muito variável quanto às manchas amarelas nas asas anteriores e posteriores. S. amoena deve ser mesmo um sinônimo, como afirmado por Evans.

2.10. Myscelus amystis amystis (Hewitson, 1867).

Erycides amystis Hewitson, 1867: 1, sem sexo, sem número de exemplares, Nova Granada, coleção Hewitson.

Myscelus flavicollis Roeber, 1925a: 158, uma fêmea, sem procedência, sem coleção.

Myscelus amystis amystis Hewitson, 1867; Evans, 1951:73, pl. 8 (genitália masculina), syn.: flavicollis Roeber, 1925.

É provável que a sinonĩnia de Evans esteja correta.

2.11. Myscelus amystis distinctus Roeber, 1925 .

Myscelus nobilis distinctus Roeber, 1925a: 159, um exemplar, Ecuador, coleção Roeber ("diante de mim").

Myscelus amystis distinctus Roeber, 1925: Evans, 1951: 73.

É provável que a identificação de Evans esteja correta.

2.12. Myscelus pardalina pardalina (Felder \& Felder, 1867).

Tamyris pardalina Felder \& Felder, 1867: 507, pl. 70, figs. 5 e 6, macho, sem número de exemplares, Nova Granada, Bogotá, Lindig leg., coleção Felder.

Agara aurora Roeber, 1925a: 92, um macho, Ecuador, coleção Roeber ("diante de mim").

Myscelus pardalina pardalina Roeber, 1925; Evans, 1951: 78, pl. 8 (genitália masculina), syn.: aurora Roeber, 1925.

A confirmação deste nome só será possivel após o exame do tipo ou a eventual designação de um neótipo. 
2.13. Phocides palemon palemon (Cramer, 1777).

Papilio palemon Cramer, 1777: 54, pl. 131, fig. F, sem sexo, sem número de exemplares, Suriname, sem coleção.

Phocides palaemonides Roeber, 1925a: 159, uma fêmea, sem procedência, sem coleção. Syn. $\mathbf{n}$.

Phocides polybius phanias Burmeister, 1879; Evans, 1952: 8, part. sinônimo incorreto:palaemonides Roeber, 1925.

A julgar pela descrição é um sinônimo de palemon palemon.

2.14 e 15. Phocides pigmalion hewitsonius (Mabille, 1883).

Erycides hewitsonius Mabille, 1883: 52, para Pyrrhopyga pigmalion (Cramer); Hewitson, Pyrrhopyga, pl. 1, fig. 3, rio Amazonas, coleção Saunders e Hewitson.

Phocides aberrans Roeber, 1925a: 94, um macho, Peru, sem coleção.

Phocides jemadides Roeber, 1925a: 94, um macho e uma fêmea, Mapiri, Bolǐvia, coleção Roeber ("diante de mim").

Phocides pigmalion hewitsonius Mabille, 1883, Evans, 1952: 13, syn.: aberrans Roeber, 1925 e jemadides Roeber, 1925.

A confirmação destes nomes de Roeber só será possĩvel após o exame dos tipos ou com eventuais designações de neótipos.

2.16. Hyalothyrus neleus pemphigargyra (Mabille, 1888).

Lignyostola pemphigargyra Mabille, 1888: 221, sem sexo, sem número de exemplares, Chiriqui, coleções Staudinger e Mabille.

Anastrus niger Roeber, 1927: 428, um macho, VI-1925, Sierra Nevada de Santa Marta, Colômbia, 1500 m, Krueger leg., coleção Niepelt.

Hyalothyrus neleus pemphigargyra Mabille, 1888; Evans, 1952: 30, syn.: niger Roeber, 1927.

É provável que a sinonimia de Evans esteja correta.

\subsection{Astraptes talus (Cramer, 1777).}

Papilio talus Cramer, 1777: 122, pl. 176, fig. D, sem sexo, sem número de exemplares, Suriname, sem coleção.

Goniurus virens Roeber, 1925a: 95, um macho, Santa Catarina, sem coleção.

Astraptes talus Cramer, 1777; Evans, 1952: 101, syn.: virens Roeber, 1925.

É provável que a sinonímia de Evans esteja correta.

2.18. Perichares quadrimaculata Roeber, 1925.

Perichares quadrimaculata Roeber, 1925b: 185, sem sexo [ ¿ ], Colômbia, sem coleção.

Este nome foi omitido por EVANS (1955), no entanto dentro do exemplar do seu livro no British Museum (Natural History) há a seguinte nota sua, a ser inserida no catálogo: "p. 257. quadrimaculata Roeber, 1925: macho, Colômbia, to be inserted as a sub-sp. of deceptus: like drina but lacking the lilacine barnds [sic] unf and unh." 
Não foi possǐvel identificar esta espécie. Só o exame do tipo ou a eventual designação de um neótipo resolverá este enigma.

\section{AGRADECIMENTOS}

Agradeço ao Dr. R. Krause do "Staatliches Museum fuer Tierkunde", Dresden, ao Dr. G. Mueller-M, da seção de Biologia, Ernst-Moritz-Arndt-Universitaet, Greifswald, ao Dr. H.-J. Hannemann, do "Zoologisches Museum der Humboldt Universitaet", Berlin, todos na República Democrática Alemã, ao Dr. P. Grootaert, Institut Royal de Sciences Naturelles de Belgique, Bruxelas, Bélgica e aos Drs. R. I. Vane-Wright e Ph. Ackery, ambos do British Museum (Nat. Hist.), Londres, Inglaterra, pelos convites e permissões oferecidos para estudar os Hesperiidae das respectivas coleções.

\section{REFERENCIAS}

BELL, E. L., 1932. Studies in the genus Phocides with descriptions of new species (Lepidoptera, Hesperiidae). Trans. Am. ent. Soc., 58:169-199, 5 pls.

CRAMER, P. 1777. De Uitlandsche Kapellen voorkomende in de drie waereld-deelen Asia, Africa an America. Vol. 2, 152 pp., pls. 97-192. Baalde, Amsterdam.

EVANS, W.H. 1951. A catalogue of the american Hesperiidae . . Part I, Pyrrhopyginae, VII + 92 pp., pls. 1-9, British Museum (Nat. Hist.), London.

EVANS, W.H. 1952. A catalogue of the american Hesperiidae. . . Part II, Pyrginae, section 1, V + 178 pp., pls. $10-25$. British Museum (Nat. Hist.), London.

EVANS, W.H. 1953. A catalogue of the american Hesperiidae. . Part III, Pyrginae, section 2, V + 246 pp., pls. 26 - 53. British Museum (Nat. Hist.), London.

EVANS, W.H. 1955. A catalogue of the american Hesperiidae... Part IV, Hesperiinae and Megathymiinae, V + 499 pp., pls. 54 - 88. British Museum (Nat. Hist.), London.

FELDER, C. \& R. FELDER, 1867. Reise der oesterreichischen Fregatte Novara un die Erde... Zoologischer Theil, Vol. 2(2), Heft 3, pp. 379-535, pls. 48 - 74, Viena.

GODMAN, F. DUCANE \& OSBERT SALVIN. 1879. Descriptions of new species of Rhopalocera from central and South America. Proc. Zool. Soc., London, pp. 150 - 155, pls. 14 e 15 .

HEPPNER, J.B. 1984. Atlas of neotropical Lepidoptera, 2, Checklist: Part 1, Micropterigoidea - Immoidea. XXVII + 112 pp., Junk Publishers, The Hague, Boston, Lancaster.

HEWITSON, W.C. 1867. Description of one hundred new species of Hesperidae. 1st. part, 25 pp. Van Voorst, London.

HORN, WALTHER \& ILSE KAHLE. 1935. Ueber entomologische Sammlungen. Teil I, 160 pp., 16 pls. Berlin-Dahlem. 
Revta bras. Zool.

MABILLE, P. 1877. Descriptions de Lépidoptères nouveaux du groupe des Hespérides. Pet. Nouv. Ent., 2:161-162.

MABILLE, P. 1883. Descriptions d'Hespéries. Ann. Soc. ent. Belg. 27, C.R., pp. 5 1-78.

MABILLE, P. 1888. Diagnoses de Lépidoptères (Hespérides) nouveaux. Le Nat., 10: 77 $78,98,108-109,146-147,169-171,180-181,221,242,254-255,265-266,275$.

PLOETZ, C. 1879. Die Hesperiinen-Gattung Pyrrhopyga und ihre Arten. Steet. ent. Ztg., Stettin, 40:520-538.

ROEBER, J. 1925a. Neue Suedamerikanische Falter (Lep.). Ent. Mitt., Berlin, 14:85-100, 156-162.

ROEBER, J. 1925b. Neue Falter. Stett. ent. Ztg., Stettin, 85:184-190.

ROEBER, J. 1926. Neue tropische Falter. Ent. Rdsch., Darmstadt, 43(4):13, 18, 22-23, $26-27,32$.

ROEBER, J. 1927. Neue exotische Falter. Intern. ent. Ztschr., Guben, 20:400 -403, 412 $414,419-421,428-430,1$ pl.

SCUDDER, S.H. 1972. A systematic revision of some of the american butterflies; with brief notes on those known to occur in Essex County, Mass. Rep. Peab. Acad. Sci., Philadelphia, 4:24-83.

STAUDINGER, O. in Staudinger, O. \& E. Schatz, 1884 - 1888. Exotische Schmetterlinge. I. Theil, Exotische Tagfalter, Vol. 1, pp. 1-333, pls. 1-30, G. Loewensohn, Fuerth.

WEEKS, A.G. 1911. Illustrations of diurnal Lepidoptera, with descriptions. Vol. 2, X + 33 pp., 33 pls., University Press, Boston. 